\title{
PROPOSING A MODEL OF SERVICE DELIVERY FOR VICTIMS/SURVIVORS OF CHILD SEXUAL ABUSE (CSA) FROM ETHNIC MINORITY COMMUNITIES IN AUSTRALIA
}

\begin{abstract}
A systematic literature review on child sexual abuse (CSA) and ethnic minority communities was conducted to help address the current gap in knowledge in the area. This article reports on one explored theme - aspects of service delivery models identified as effective, as the basis for developing a model for Australia. The findings indicate two key aspects of effective service delivery: (i) models should be ecologically grounded to the specific cultural context, and (ii) individuals should be placed at the centre of intervention so their needs and experiences are not 'boxed in' by their ethnicity. The model proposed for the Australian context is guided by these two key principles. Service delivery models are important because they help guide practice and standardise treatment and support in the field. However, the model proposed here also values flexibility, cultural competency, a strengthsbased approach, an empowerment-based approach, intersectionality, and a feminist framework. These model elements are particularly relevant in relation to the family cohesion and support that collectivist cultures offer; whether, to what extent, and how they act as a risk or protective factor, or even both, is best determined by the victim/survivor. The utility of the model for practitioners and clients requires rigorous evaluation.
\end{abstract}

\section{Keywords}

Child sexual abuse (CSA), ethnic minorities, service delivery models, cultural competency, collectivism, selfdetermination 


\section{PROPOSING A MODEL OF SERVICE DELIVERY FOR VICTIMS/SURVIVORS OF CHILD SEXUAL ABUSE (CSA) FROM ETHNIC MINORITY COMMUNITIES IN AUSTRALIA}

\section{Introduction}

\section{Background, research questions, and significance}

In 2013-2017, the Australian Government spent nearly \$1b on the Royal Commission into Institutional Responses to Child Sexual Abuse ${ }^{1}$; reflecting Australia's value for child safety and commitment to help protect children from sexual harm. Current figures from the Australian Institute of Health and Welfare (AIHW) show that 7,171 children had substantiated reports of child sexual abuse (CSA) as the primary type of maltreatment in 2015-2016. However, national data on whether CSA varies cross-culturally - beyond the distinction between Indigenous and non-Indigenous Australians - is not collected and therefore available (Mathews et al., 2016). This is consistent with a broader trend, both in Australia and elsewhere, that research on CSA and ethnic minority communities is scant; widely acknowledged in the field of child protection (Bromfield \& Arney, 2008; Burke \& Paxman, 2008; Cashmore, Higgins, Bromfield, \& Scott, 2006; Futa, Hsu, \& Hansen, 2001; Tishelman \& Geffner, 2010; Sawrikar, 2017). There is some evidence from a number of countries that children from some cultural groups are over-represented in child protection systems, but there also is no consensus as to why this is the case (Katz \& Connolly, 2017).

To help address the gap in national (and international) research and knowledge, a systematic literature review was conducted. It explored six themes (agreed upon by eminent scholars in the field of child protection in Australia; see 'Acknowledgments'): (i) community awareness of CSA among ethnic minorities, (ii) prevention of CSA among ethnic minorities, (iii) issues related to disclosure of CSA among ethnic minorities, (iv) the treatment needs of victims/survivors of CSA from ethnic minority communities, (v) possible service delivery models for victims/survivors of CSA from ethnic minority communities in Australia, and (vi) current national service provision to victims/survivors of CSA from ethnic minority communities in Australia.

This article reports the findings of theme five, thus focusing on service delivery models (the results of the other themes are reported elsewhere; see Sawrikar \& Katz, 2018, 2017 a, b, c). Specifically, it addresses three research questions: (1) what are all the service delivery models currently available in the relevant body of

\footnotetext{
${ }^{1}$ https://www.childabuseroyalcommission.gov.au/
} 
literature? (2) what characteristics or aspects of service delivery models are identified as effective? and (3) what is a possible service delivery model that could be used by service agencies in Australia?

In addressing these three research questions, this article begins to contribute to national capacity-building and thus is a significant contribution to the literature. When themes are transferable to comparable Western multicultural countries such as the US, UK, Canada, and New Zealand, it also contributes to the international literature. Overall, they help address the issue that a lack of a service delivery model could leave practitioners unaware of and unsystematic in their approach to the variables they might need to consider when assessing and conceptualising an appropriate clinical response to victims/survivors of CSA from ethnic minority communities and their families. This in turn could leave already vulnerable ethnic minority victims/survivors - from the trauma of CSA itself as well as compounded issues to do with racism (see Sawrikar \& Katz, 2017 a, b for more information on these issues, which are outside the scope of this paper) - at further risk of poor quality service delivery.

\section{Defining the client group}

Ethnic minority communities in Australia have a migration history and are minorities in at least one of the four main dimensions of ethnicity - race, language, culture, and religion (O'Hagan, 1999). As such, they are differentiated from their Indigenous (Aboriginal and/or Torres Strait Islander) and Anglo Australian (Saxon and/or Celtic) counterparts, and tend to come from non-English speaking backgrounds (NESBs) and countries high on collectivism (see 'Theoretical framework' for more information). The term 'ethnic minorities' includes refugees and asylum seekers but humanitarian migrants have additional needs and experiences to the wider category, which includes those who have chosen to migrate, are skilled migrants, and/or have been in Australia for several generations (Allimant \& Ostapiej-Piatkowski, 2011). The term 'culturally and linguistically diverse' (CALD) is used in research, practice, and policy in Australia, but has not been used here because it only highlights culture and language, to the exclusion of race and religion, as factors relevant to this group (Sawrikar \& Katz, 2009).

\section{Theoretical framework}

As stated earlier, ethnic minorities tend to be high on collectivism. 'Collectivism' is a cultural stance that views the basic unit of society to be the family, and is differentiated from 'individualism' which views the individual to be the basic unit of society (Hofstede, 1980). More specifically, collective cultures value social order, 
harmony, support and roles; in collective cultures the family provides security in exchange for loyalty and obedience; inequality (usually based on age and gender) is seen as appropriate and acceptable; and members tend to be more homogenous as deviations from the norm are not tolerated as greatly (Bond, 2002; Triandis, 1990). In contrast, individualistic cultures value independence, autonomy, initiative and uniqueness; emphasise that individuals have the right and responsibility to look after themselves; stress horizontal relationships based on equality; and tolerate deviations from the norm to a greater extent (Bond, 2002; Triandis, 1990).

According to Hofstede (2001), the US, Australia, UK, Canada, and New Zealand, all rank (in order) highest on individualism. Western European countries rank next highest, and the remaining parts of the world (e.g. East Europe, Asia, South America, Middle East, Sub-Saharan African, etc.) all rank more highly on collectivism. The implication is that many ethnic minorities in Western countries like Australia originate from non-Western countries high on collectivism.

Moreover, cultural values from the country of origin remain pervasive, even for well-established migrant groups and across generations, because the extensive literature on acculturation shows that norms, values, beliefs, and traditions do not simply disappear into a 'melting pot' in the new country. Migrants continuously manage two conflicting needs - the need to preserve the culture of origin and the need to adapt to the new culture (Berry, 1980) - some in response to changing social cues. For example, they are affected by whether the person speaks or understands their native language, whether they were born overseas, their age, whether they are visibly different, how much racism and discrimination they experience, whether the local area they reside in is accepting of multicultural diversity, and national and international political discourse (e.g. vilification of Muslims, derogatory terms for refugees and asylum seekers such as 'boat people', etc.) (Sawrikar \& Katz, 2010; Sawrikar \& Katz, 2009; Sawrikar \& Hunt, 2005). Thus, the acculturation of cultural values such as that of collectivism is dynamic across the lifespan.

The theoretical framework of individualism/collectivism is being used here is because it is critical for understanding the daily lived experiences and social structures of, and therefore CSA in, ethnic minority communities. Specifically, collectivist cultures place greater value for family and community over the needs of individuals, and therefore in the context of CSA the needs of victims may be de-prioritised to the needs of the family, most especially the need to protect the family's reputation or face.

Collectivist cultures also overtly accept gender inequalities. Thus, the father is usually seen to be the head of the family, has most of the power within the family, and in turn is most associated with the family's 'face' or 
reputation. Allegations of CSA against fathers or other senior males in these cultures are therefore perceived as accusations against the whole family, and in order to save 'face' it is often the accuser and/or victim that is excluded by the family rather than the alleged abuser.

Importantly, the theoretical framework of individualism/collectivism is not being used to minimise the importance of family, reputation, patriarchy, silence, and exclusion among individualistic groups (which does all occur), but to highlight their centrality among collectivist ones. This is necessary when attempting to identify how best to protect children in these communities; it provides a culturally relevant 'lens' for making sense of their needs and experiences. It therefore aligns with social constructionism (that perceived reality is constructed by personally meaningful and relevant variables), cultural relativism (that not all cultures can or should be compared, as if they all occur along comparable dimensions), and emic methodologies (that 'insider' perspectives matter; both authors are ethnic minorities).

Although the theoretical framework is seen as useful, it is still important to point out that they are just generalised stereotypes, which occurs because cultures tend to be heuristically categorised as one or the other whereas in fact all cultures exist on a continuum of individualism and collectivism. Thus, these heuristic categories should not be definitively relied on; they need to be used with vigilance, ensuring that the beliefs comprising the stereotypes are accurate, do not perpetuate harm or disadvantage to groups, and are questioned when applied to individual situations (Sawrikar, 2017). This is particularly critical for clinical practice because stereotyped assumptions about culture can result in inappropriate and even harmful outcomes (Owusu-Bempah \& Howitt, 2000).

Ultimately, there are many ways of examining the role of culture, which can broadly be defined as any "timehonoured tradition that is considered normal, acceptable, and/or of value" (Sawrikar, 2017, p. 18). As Korbin (2008) puts it, "culture is constantly changing defying easy definitions or the construction of a library-like catalogue of behaviors and values and instead demanding finely nuanced contextual understandings" (p. 122). Thus, the broad or macro-level conceptualisation of culture used here - the distinction between individualism and collectivism - is seen as necessary but not sufficient for understanding CSA among ethnic minority communities.

\section{Method}

\section{The PRISMA process}


The methodology for obtaining the relevant body of literature was informed by the Preferred Reporting Items for Systematic Reviews and Meta-Analyses (PRISMA) process. See Figure 1 for the PRISMA flow diagram (Moher, Liberati, Tetzlaff, Altman, \& The PRISMA Group, 2009).

\section{Figure 1: PRISMA flow diagram}
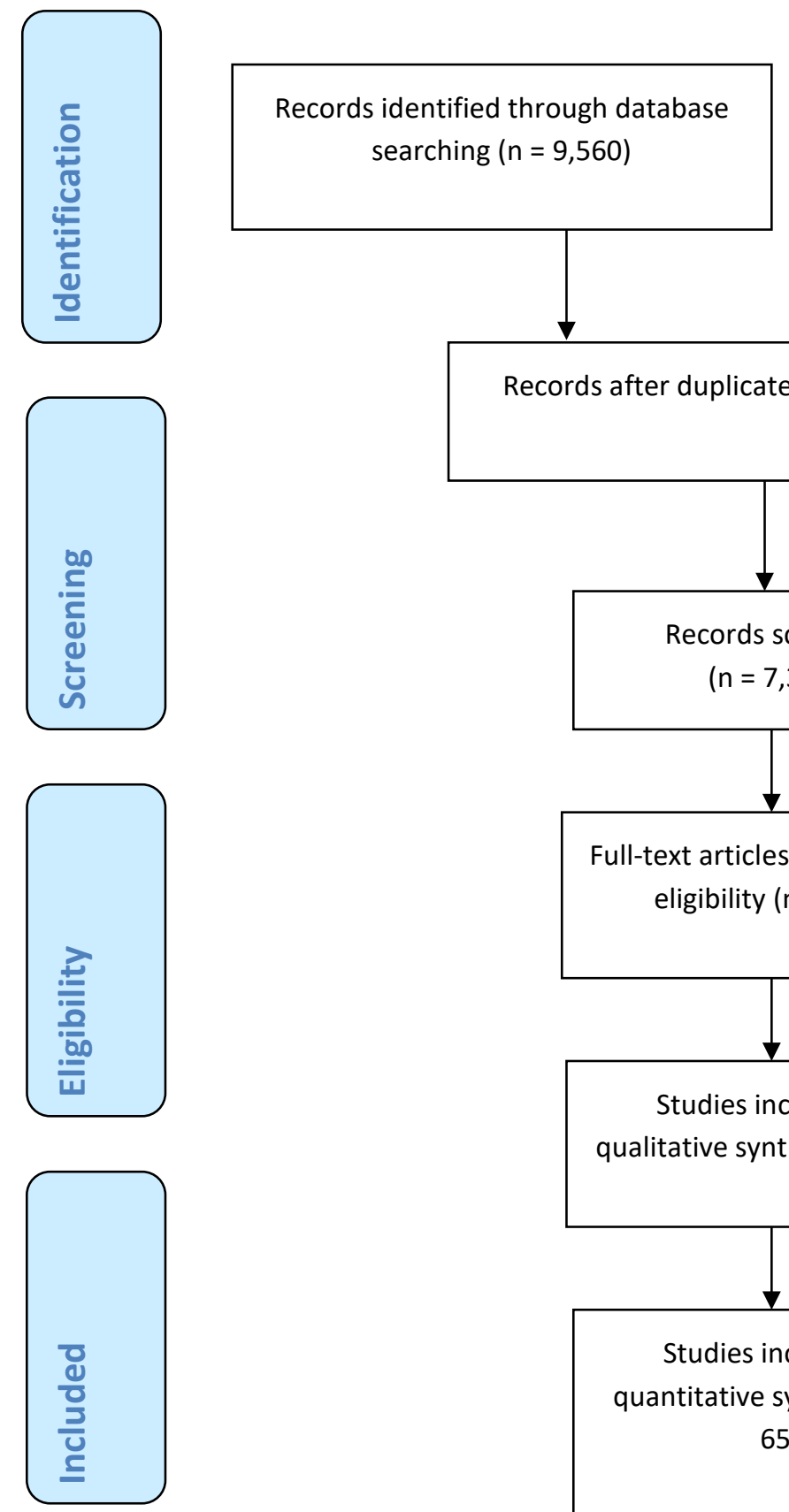
Additional records identified through other sources $(n=0)$

Records after duplicates removed $(n=7,363)$

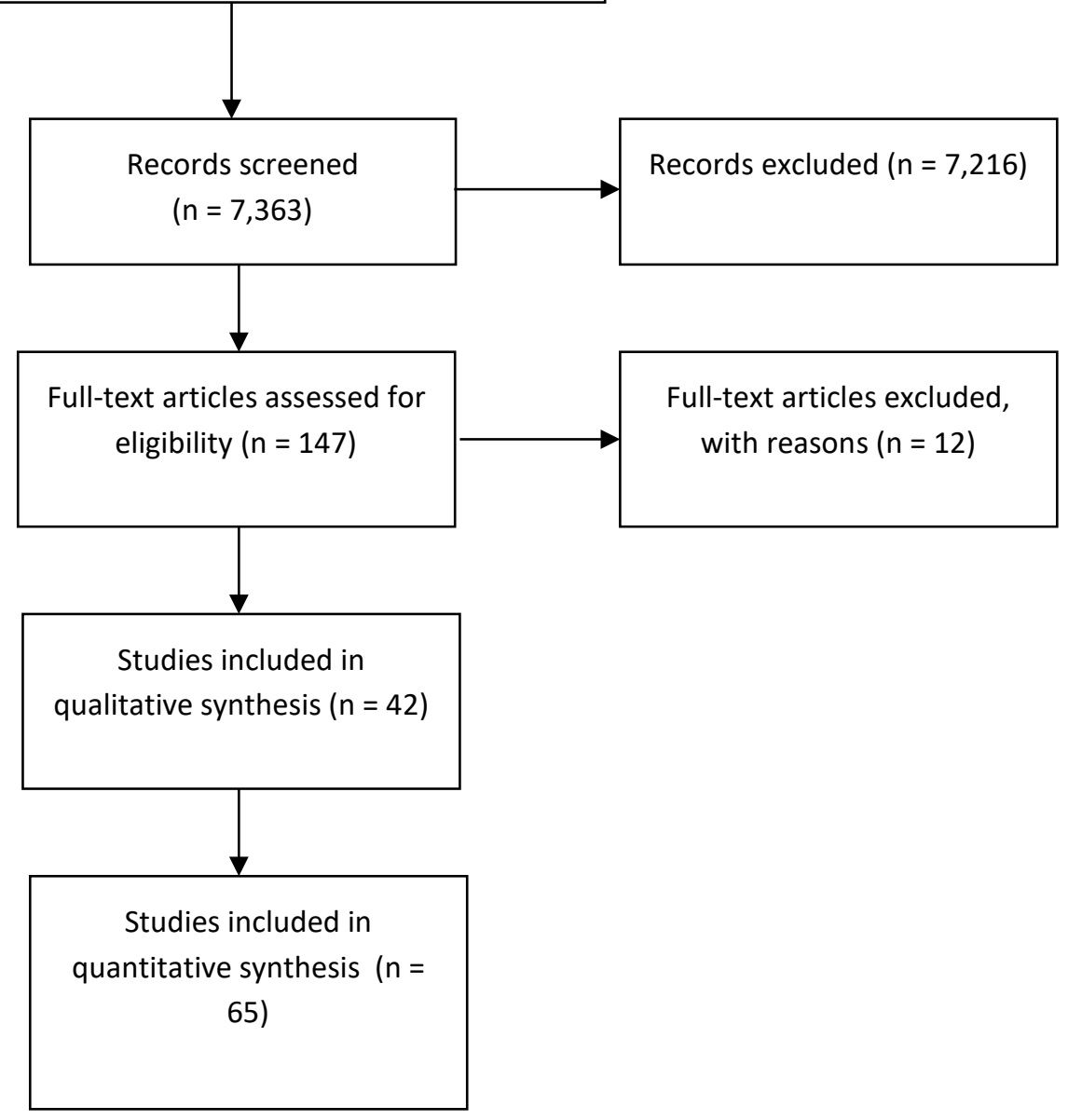


The study first sourced literature from relevant databases. These included: Australian Public Affairs Full-Text, Health and Society Database, Informit Family and Society Collection, Informit Health Collection, Informit Humanities and Social Sciences Collection, Multicultural Australia and Immigration Studies, ProQuest Family Health, PsycINFO, Scopus, Social Services Abstracts, and Sociological Abstracts.

It then combined a series of search terms related to the two keywords - 'child sexual abuse' and 'ethnic minorities'. Search terms used for the first keyword included: sexual abuse, sexual harassment, sexual exploitation, molestation, rape, indecent assault, sexual violence, and gender violence. Search terms used for the second keyword included: culture (cultur*), race (rac*), culturally and linguistically diverse (CALD), nonEnglish speaking background (NESB), ethnic minority/ies (ethni*), migrant, immigrant, refugee, asylum seeker, of color/colour, and community.

It then limited searches to 2000-2016 to ensure only recent literature was included (however seminal research was not excluded and was sourced from the reference lists of the newer articles). Where available, the search was also limited to articles in English, peer-reviewed, and with the full-text available. These criteria led to 9,560 results. Duplicates were then removed $(n=2,197)$, reducing the number of records to 7,363 . They were then screened for their relevance to the six themes of the larger review based on their title, keywords, and abstract. Most of the literature was excluded at this stage; although the Boolean logic of 'AND' had been used to combine search terms related to the two keywords, the searches mostly did not yield literature specifically related to sexual abuse experienced in childhood by someone from an ethnic minority background (i.e. ethnic minorities in Western countries or about non-Western countries). Most commonly, articles were excluded because they related to sex offenders and/or recidivism, sexual risk behaviour but not sexual abuse, elder (sexual) abuse, military culture, and substance abuse, men who have sex with men, and women who have sex with women but not sexual abuse. In total, 6,715 records were deleted at this stage. Of the 648 remaining articles, the full text of 20 was not available, leaving 628 records. Of these, 421 related to sexual violence, gender violence, domestic violence (DV), intimate partner violence (IPV), sexual exploitation/trafficking, and sexual harassment among adults rather than children or survivors of CSA in ethnic minority communities, or were about violence that involved children but not CSA (such as witnessing DV); these too were excluded.

Literature was also excluded if it only pertained to Native Americans and African Americans to address $a$ priori issues to do with the transferability of findings from Indigenous groups in other Western countries and/or 
minority groups not represented in Australia. Literature only pertaining to Latina/Hispanic Americans was also excluded on the grounds that they form a large and significant minority group in the US and so their needs and experiences could be quite specific to that context. Indeed, Kenny and McEachern (2000) found that "many studies (about CSA in minority communities in the US) utilised Asians in their homelands and AsianAmericans, while research examining Hispanics utilised only Hispanic immigrants to the United States" (p. 906). However, literature that addressed groups in addition to Native, African, and Latina/Hispanic Americans for example, Asian-Americans (because Asians are highly represented in Australia) - were included in this review, so the former groups have not been wholly disregarded. This helps ensure that the experiences of all minority groups are captured to some extent in this review.

Applying all these criteria left a total of 135 records eligible for full review. Of these, 42 primarily used qualitative methodologies, 65 primarily used quantitative methodologies (including meta-analyses), eight used a mixed-methods approach (counted under the 'Quantitative' records), and 28 used secondary analyses (e.g. literature reviews, commentaries, etc.). As the secondary analysis records did not use either qualitative or quantitative methodologies, they do not appear in the flowchart. The broader literature on 'child maltreatment and ethnic minority communities' and 'family violence and ethnic minority communities' (from previous research conducted by the authors) were also drawn upon where relevant. Only the articles relevant to the theme of service delivery models have been cited here.

\section{Strengths and weaknesses}

The methodology of the review was systematic, comprehensive, and exhaustive. It also identified all themes in the reviewed literature thereby exhausting them, as well as describing all literature (within word limits) relevant to each theme. These themes have been described in the form of a narrative review (under 'Results'), and thus thematic analysis is merged with description. Nevertheless, some methodological limitations need to be noted.

The first is that the body of literature on CSA and ethnic minorities is small (as already stated), and not all of the studies are based on large samples or rigorous methodologies. The literature on service delivery models within that is even smaller. This limits being able to offer evidence-based principles of best practice regarding service delivery, with only suggestions being able to be offered. Still, literature in Western contexts cannot and should not be used to make sense of the needs and experiences of ethnic minorities, as several researchers point out (Baker, Gleason, Naai, Mitchell, \& Trecker, 2013; Chen \& Chen, 2005; Chien, 2013; Elbedour, Abu-Bader, 
Onwuegbuzie, Abu-Rabia, \& El-Aassam, 2006; Gilligan \& Akhtar, 2005; Mildred \& Plummer, 2009; Reavey, Ahmed, \& Majumdar, 2006; Sil \& Soo, 2008; Usta \& Farver, 2010; Wang \& Heppner, 2011). Overall, there is a call for much greater research in the area of CSA in ethnic minority communities.

Secondly, the review has grouped several different races, cultures, languages, and religions together, falsely homogenising each of their needs. Fontes (1993) calls this 'ethnic lumping'. It usually occurs because of small sample sizes in research for each group, warranting combining them to be able to say something more representative of the larger category. There is some merit in this - by virtue of being a minority, various groups do have 'a something' in common, making results for one group reasonably generalisable to another. It also has merit at a broad cultural level - many ethnic minority groups are culturally collectivistic, so again cultural trends are likely to generalise from one group to another. Where it has limitations, however, is at the more nuanced and detailed level of culture, language, and religion. Traditions, beliefs, norms, and values about CSA will not necessarily transfer to another. These limitations must be acknowledged, and addressed where possible, as part of ethical conduct in research. Overall, however, the inclusion of articles from diverse backgrounds is a global approach consistent with and required for issues that address multiculturalism.

Another issue is that much of the literature is about ethnic minority groups in other Western countries, most especially the US and UK, posing issues for the transferability of findings to the Australian context. This issue was somewhat addressed by excluding literature that related solely to groups not represented in Australia (e.g. Native and African Americans), however caution still needs to be exercised. Reasonable comparisons can be made with other Western countries like the US, UK, Canada, and New Zealand, however they do have different 'ethnic profiles' to Australia, with different specific groups and sizes comprising their migrant community, as well as reasons for their migration to these countries, which all affect their socio-cultural and political standing there (Sawrikar \& Katz, 2008).

Finally, there is the issue that themes identified in the literature on non-Western countries of origin may only partially help explain the needs and experiences of ethnic minorities in Western countries because the migration experience can interact with cultural factors in such a unique way that literature from 'back home' could become limited or even irrelevant. Moreover, literature from non-Western countries of origin was not equally represented; most was about East Asians and South Asians, sub-Saharan and South Africans, and Arabs and Jews, so the experiences of other groups are even less well documented. 


\section{Results}

\section{Currently available service delivery models}

Despite that the body of literature on CSA and ethnic minorities is small, six models were still identified (briefly described below; their key elements are expanded on in research question 2). This is proportionately seen as a positive step toward knowledge-building in the area because it indicates that a more analytical, rather than simply descriptive, approach to theory is garnering some momentum. Having said that, the models described here are all theoretical rather than clinical, and so do not directly address the first research question of models that can be used to guide the service provided by practitioners (and therefore their utility in terms of client outcomes).

The first four of these theoretical models are based in non-Western/collectivist contexts and therefore may not have transferability to the Australian context, but are still being reported on because the aim of the first research question was to identify all available models in the current body of literature. The remaining two models are about ethnic minorities in Western/individualistic countries and therefore do have more transferability to ethnic minorities in Australia but are only based on one ethnic group in the US (South Asians), highlighting the overall need for much further work on both theoretical and service delivery models for this group of victims/survivors.

Models in non-Western countries of origin

\section{Model 1 - 'Deployment-focused model' developed in Bali}

Lesmana, Suryani, and Tiliopoulos (2015) explored cultural considerations in the treatment of mental illness among sexually abused children and adolescents in Bali, Indonesia, and then proposed a 'deployment-focused model'. They argue that while it has been developed in Bali, it could be used for other collectivist cultures too.

The main premise of the model is that therapy "in individualistic societies often focusses on a single disorder, in a linear sequence, such as exclusively treating PTSD or depressive symptomatology, usually through some form of cognitive therapy" (p. 111). However, in collectivistic societies there is an additional need to "contextually consider the specificity of client needs (including) cultural elements present in the reporting of child sexual abuse cases" (p. 111). 
In this regard, they propose a more "innovative, biopsychosocial, psychotherapy" (p. 111), which they describe as "holistic, sensitive, and culturally competent" (p. 114). This is because "it mobilises modern psychotherapeutic techniques and medication, while respecting and responsibly utilising traditional Hindu health practices and religious beliefs of the focal human geography, specifically mediation and visiting a Balian spiritual healer/guide, combined with community workshops held in temples and other religious holy places" (p. 114).

\section{Model 2 - 'Transactional and ecological model of coping' in Taiwan}

Wang and Heppner (2011) qualitatively explored the experiences of 10 female Taiwanese childhood sexual abuse survivors to broaden understanding of the post-abuse coping process in a Chinese sociocultural context. They used a feminist paradigm, and developed a transactional and ecological model of coping, "inspired by the ecological framework proposed by Bronfenbrenner (1977), in which individual developmental processes are influenced by interactions within and between (1) the 'microsystem' (the immediate setting, such as families), (2) the 'mesosystem' (the system of microsystems, such as peers), (3) the 'exosystem' (an extension of the mesosystem, such as the mass media and government agencies), and (4) the 'macrosystem' (the overarching institutional patterns of the culture)" (p. 395).

Their model argues that factors related to the immediate 'self' (i.e. psychosocial adjustment to CSA) interacts with their 'relational context' (i.e. family, friends, romantic partners, others, and the perpetrator), as well as their 'sociocultural context' (i.e. norms, values, customs, and sociopolitical history). These authors also call for more feminist participatory action research (PAR). Feminist and ecological approaches to knowledge-building are seen to value "holistic and naturalistic description of the entire child sexual abuse phenomena and cultural contextual factors" (p. 394). They are also seen to give victims/survivors a voice, broaden understanding, and empower survivors. Overall, this is a theoretical model but it could also be used to frame service delivery.

\section{Model 3 - 'Contextually sensitive model of intervention' in Palestine}

The model of intervention proposed by Shalhoub-Kevorkian (2000) is intended to be an alternative to those used in the West when dealing with female victims/survivors of sexual abuse in collective, non-Western societies. The model contains five essential elements for service support organisations: (i) establishing dialogue (i.e. breaking the silence), (ii) mapping and activating potential resources, (iii) securing acknowledgment of the 
abuse and commitment to its cessation, (iv) intensive follow-up and ongoing assessment, and (v) summative evaluation.

The key element of this model is that, against the "potent gender bias" (p. 630) in Palestinian-Arab society, women are still able to activate culturally-relevant resources to protect themselves in the present and future (including their own life). Specifically, "it challenges the belief in Western societies that the state is the sole agency capable of dealing with this crime through formal legal interventions, which is an extreme form of individualism (for) Palestinian society" (p. 631).

Thus, this model is culturally sensitive and relevant because it does not impose Western/individualistic conceptualisations of protection and justice, it centrally uses the collectivist value for family and community and their dynamics, and it asks for a re-appraisal "of 'honour' (which can lead to killing the victim) to signing a legal agreement (indicating the caregiver's commitment to stop the abuse) comparable to giving one's 'word of honour' in Arab society" (p. 631). Together, these can help block or minimise the exclusion the victim would otherwise suffer.

\section{Model 4-Self-determined risk and protective factors of CSA in Kenya}

Based on qualitative work with professionals, Plummer and Njuguna (2009) identified a list of protective and risk factors regarding CSA in Kenya (see Table 1). These were not identified as a service delivery model or framework by the authors, but could still be used for this purpose. Importantly, these protective and risk factors were identified by Kenyan professionals for the Kenyan context; the extent to which they may transfer to ethnic minority communities in Australia would require thorough investigation.

\section{Table 1: Protective and risk factors regarding CSA in Kenya}

\begin{tabular}{|l|l|}
\hline \multicolumn{2}{|l|}{ Protective factors } \\
\hline Individual level & Modesty (reserved clothing) \\
\hline Family level & Family structure (e.g. mothers at home to supervise) \\
\cline { 2 - 2 } & Guidance and supervision (specific ways supervision is provided) \\
\hline Cultural group level & Religion (reference to faith) \\
\cline { 2 - 2 } & Separation (of males and females to prevent abuse) \\
\hline
\end{tabular}




\begin{tabular}{|c|c|}
\hline & Value children (children are well-loved and a future resource) \\
\hline & Harsh deterrents (severe outcomes to discourage offenders) \\
\hline & Sex and gender taboos (expected gender roles) \\
\hline & $\begin{array}{l}\text { Importance of virginity (to protect dowries, monetary loss, family pride, and rituals that } \\
\text { celebrated virginity until marriage) }\end{array}$ \\
\hline \multicolumn{2}{|l|}{ Risk factors } \\
\hline Individual level & Individual risks (e.g. divorce, homelessness, being an AIDS orphan) \\
\hline \multirow[t]{5}{*}{ Cultural group level } & Gender roles (for male and female children that may place them at greater risk of CSA) \\
\hline & Patriarchy (male dominance and perceived superiority) \\
\hline & $\begin{array}{l}\text { Culture of silence (tribes protecting their own social norms by discouraging discussion } \\
\text { of sex or abuse) }\end{array}$ \\
\hline & Sexual norms (ideas about sex and sexuality including FGM and early marriage) \\
\hline & Role of children (low status and value, not permitted to speak, lacking power) \\
\hline \multirow[t]{3}{*}{ External factors } & $\begin{array}{l}\text { Foreign influences (Western values, pornography, and internet seen as increasing } \\
\text { incidence of CSA) }\end{array}$ \\
\hline & Social change (upheavals e.g. war, refugee camps, urban migration) \\
\hline & Poverty \\
\hline
\end{tabular}

Models about ethnic minorities in Western countries

\section{Model 5 - 'Resilience-based trauma model' for South Asian Americans}

In her proposed model, Singh (2009) argues that there are two core constructs at the heart of a "culturally relevant model of resilience for South Asian women survivors of sexual abuse (in the US)” (p. 369). These are gender and acculturation.

In the counselling context, assessing an individual's acculturation level (traditional, bicultural, or Westernidentified), "allows their narratives of trauma and resilience to be spoken and understood more fully" (p. 370). For example, if her acculturation level is 'traditional', family and community are likely to be sources of support, and therefore provide resilience that can protect her from racism from other groups and psychological distress 
incurred from having to manage multiple identities. Having said that, "family and community can also serve as a source of trauma" (p. 369).

A traditional acculturation level is also associated with the extent to which a woman "ascribes to patriarchal norms and values" (p. 370), and in turn, with how much she is likely to remain silent about her abuse. While silence "encourages the development of trauma symptomology" (p. 370), it can also encourage the development of resilience strategies. This is because, "through silent rebellion, they develop self-reliance and mental strength, such that silence is no longer just a survival mechanism, but a testament to resilience, equated with inner power" (Poore, 2000, p. 9, cited in Singh, 2009, p. 365).

Given that healing "includes the ability to make meaning of one's experience" (p. 370), this model enables the victim/survivor to determine what is a risk factor and what is a protective one. Singh and colleagues (Singh, Hays, Chung, \& Watson, 2010) extended these core ideas by later proposing a phenomenological model of resilience, in which the meaning of CSA for South Asian victims/survivors is driven foremost by their South Asian context (gender socialisation, family image, influence of ethnic identity, and acculturative stressors) and resilience (use of silence, sense of hope, South Asian social support, social advocacy, and intentional self-care). More broadly, these models of resilience heed the call to "expand from traditional pathology-based approaches to a wellness- or strengths-based approach" (Singh, 2009, p. 366).

\section{Model 6 - 'Idealised cultural identities model on help-seeking' among South Asian Americans}

Similar to Singh (2009), Kanukollu and Mahalingam (2011) acknowledge the important roles of level of acculturation, adherence to traditional gender ideology, and awareness of CSA in their model of help-seeking for South Asian Americans. However, they additionally argue that internalising the myth that some minorities, like South Asians, are 'model minorities', can also negatively impact on help-seeking behaviour.

Historically, South Asians were permitted entry to the US based on their high education and therefore professional contribution to the labour force. These trends helped create the 'model minority myth' or stereotype. In addition to compromising mental health per se, because "the price of success was one's ethnicity and identity" (Pedraza, 2006, cited in Kanukollu \& Mahalingam, 2011, p. 222), this stereotype can also lead to "glossing over mental health issues (caused by CSA) within the South Asian community" (p. 223). That is, "the pressure to be a model minority member in society likely plays a role in silencing their experiences of child 
sexual abuse" (p. 223). Thus, these authors argue that the more minority members internalise the myth themselves, and have an 'idealised cultural identity', the less likely they are to seek help.

\section{Characteristics of effective service delivery models}

Using ecological approaches

The results of the review highlight the importance of designing models that are ecologically framed (Al-Fayez, Ohaeri, \& Gado, 2012; Fontes, 1995, cited in Chien, 2013); valuing the specific cultural context that victims/survivors of CSA are shaped by and live everyday life in, and using its attributes to design contextually relevant therapeutic intervention. Indeed, "Petersen et al. (2005) argue that distal influences, such as community values, are at least as important as proximal influences such as individual and family factors in understanding child sexual abuse, because whether a culture is more individualistic or collectivist has an effect on relationships, responsibility, choices, self-construals, attitudes, conflict, and values (Triandis, 1995). (More importantly), the ecological model shifts thinking away from what the potential victim, or even his/her family should do to ensure health or safety, toward a greater recognition of social structures that may promote abusiveness, through overt or covert means" (Plummer \& Njuguna, 2009, p. 526).

According to Kenny and Wurtele (2012) "an ecological approach to child sexual abuse prevention is (also) highly compatible with a public health framework. In the public health field, consensus has emerged that the etiology of many health problems arise from multiple levels and that interventions focused on a single level of influence are limited. An ecological approach emphasises that behaviours happen within a context and examines the bidirectional transactions between human beings and the systems in which they interact. (Thus), based on Bronfenbrenner's (seminal) ecological model, prevention is increasingly moving from individual-level activities to systematic strategies to change macrosystem characteristics like legislation and social norms" (p. 362).

\section{Not stereotyping}

The results also point to the importance of valuing the victim/survivor of CSA as a person first, and not as a person from an ethnic minority community first. While race and other markers of ethnicity like language and religion should not be ignored, they also should not be used to 'box' or stereotype the needs of individuals.

People and groups within the ethnic minority community in Australia vary greatly among themselves, according to their length of residency, type of visa or citizenship, knowledge of Australian systems, English proficiency, 
accent, capacity, interest, and willingness to engage with people from other cultural groups, experiences of racism and discrimination, and personal endorsement of individualistic or collectivist values (Nelson, Dunn, \& Paradies, 2011). Thus, these factors can impact on the nature and extent of intervention that may be required for a victim from this group. However, these variables all interact with the person's unique traumatic experience/s. Thus, it is really only the victim/survivor who knows what they need in the service context.

As Allimant and Ostapiej-Piatkowski (2011) put it, "fundamentally, the most supportive approach is one in which the worker can demonstrate that she values the woman's knowledge and life experiences" (p. 10). Similarly, Nesci (2006) says, “don’t look at the needs of a woman in terms of her ethnic or religious background. Work with her to find out what her particular needs are and what her understanding is of the service. Ultimately, no two women are the same nor are their needs, whether she is from English or non-English speaking background, a woman with disabilities, a young woman or an older woman, a woman with children or without children etc. With limited resources, assessing the woman's situation against a set criteria may be necessary - (but) perhaps this could go hand in hand with engaging the woman and supporting her to be in control of her life" (p. 44).

\section{Using strengths-based approaches}

The findings also demonstrate the importance of using a strengths-based, rather than deficit-based, approach to the treatment of CSA (Vermeulen \& Greeff, 2015; Singh \& McKleroy, 2011). Meinck, Cluver, Boyes, and Mhlongo (2015) note that "protective factors against abuse within both developing and developed world samples have been severely understudied" (p. 100). Thus, more research on culturally specific concepts of resilience and culturally specific protective factors are required worldwide.

\section{Self-determination regarding whether family cohesion is a risk or protective factor}

The nascent literature indicates that perhaps the most important issue to consider, in terms of whether it is a risk or protective factor, is family cohesion in collectivist cultures. Several authors note its capacity to act as both.

Lesmana et al. (2015) say that "the Balinese culture possesses a pervasive spiritually based system that emphasises relationships to foster solidarity and cooperation within the nuclear family, the extended family, and the community, (so) family and community are tightly enmeshed and interdependent. (It is also believed) that children are reincarnated ancestors whose souls are physically reborn into the bodies of infants. Since the souls 
of ancestors are highly revered and worshipped, it follows that their reincarnated physical form is similarly honoured and worshipped. Such child worship, embedded in religious beliefs, forms the basis for the degree of respect, sustained attention, love, and devotion shown to children, and appears to be a significant contributor to the formation of secure childhood attachments to primary and other caregivers" (p. 112).

Li, Ahmed, and Zabin (2012) conducted a rigorous quantitative study examining the association between CSA and adverse psychological outcomes among 4,084 youth (aged 15-24 years) in Taipei. They say, "most of the Asian area, including Taiwan, is strongly influenced by Confucianism despite industrialisation. Confucianism is an ethical and philosophical system, consisting of five 'cardinal relationships', (thus) specific responsibilities and roles are prescribed among family members. (That is), the family plays a central organising role in people's lives and provides individuals with security, support, and strong interdependent bonds which may protect child sexual abuse victims from adverse outcomes" (p. S46). This assertion is based on their finding that "Confucian values regarding family were negatively associated with depression. It suggested that young people who believe that family is an important source of support and that family members should take care of each other were less likely to be depressed" (p. S49-50).

Singh and McKleroy (2011) similarly say that examining the protective role of family and its support helps avoid relying on "the literature on resilience of trauma survivors from a Western, White approach that focusses on individual as opposed to relational processes of resilience" (p. 35). This was based on a study exploring the resilience of transgender people of colour in the US who have survived various traumatic life events (not just CSA).

Tummala-Narra (2007) states, "cultural attitudes and ideals play an important role in fostering and helping to maintain resilience in the aftermath of trauma. A particular trait or circumstance that is seen as promoting resilience in one cultural context may actually be seen as a liability in a different cultural context ... In cultures with a collectivistic orientation, an individual's reliance on coping strategies that exclude family wishes and obligations as guides for coping with interpersonal violence, such as rape or domestic violence, can be experienced as isolating to the individual and stressful to both the individual and his/her communities of reference" (cited in Okur, van der Knaap, \& Bogaerts, 2016, p. 108-109).

Finally, in the literature review of a nationally representative study of child maltreatment reports in Israel conducted by Sulimani-Aidan and Benbenishty (2013), the authors report that "familism is regarded as a 
commitment to provide an emotional support system for family members. It places great emphasis on dependency and reliance on others, as well as on family unity, with a sense of obligation among family members to care for all members, especially children. Familism characterises many Hispanic, Arab, and African American communities. (For example), African Americans who live in close proximity to their extended families report greater life satisfaction, and kin play an important role in the care of young children and in the children's positive development. (Indeed), Coohey (2001) found that familism was a protective factor against child abuse for both Hispanics and non-Hispanic Whites" (p. 34).

However, Stewart (2005) importantly points out that "beliefs about the protective effects of 'collectivist'/ sociocentric cultures may serve to hide the context of suicidal experiences of many people. Idealised images of extended family groups and 'communal living' may well have the effect of hiding the occurrence of child abuse and domestic violence, making it more difficult for victims to disclose and seek help. When the family is the source of the problem, or is unable to solve the problem and discourages the seeking of outside help, the negative effects of a 'close community' may be compounded. In this context, "when taken to extremes, protective factors transmute into risk factors" (Fry, 2000, p. 158)" (p. 11). In other words, "there is a tendency in the cross-cultural suicide literature to frame particular variables such as 'collectivism' and 'religiosity' as potentially protective factors for suicide, (but this is) an assumption that requires interrogation" (Stewart, 2005, p. 7).

That familism can be both a risk and protective factor was noted by other authors too. Sulimani-Aidan and Benbenishty (2013) said, "the concept of familism and the centrality of the family in Arab (Israeli) culture, while a protective factor against child maltreatment, have also been associated with increased levels of maltreatment and attitudes that support using violence against women and children” (p. 34).

Boakye (2009) says, "the sense of collective interest strongly felt and shared in (collectivist) African families, may not itself be the problem, but rather it is when this shared sense of collective interest is deployed indiscriminately so that negative practices (e.g. female genital mutilation; FGM) and antisocial behaviours or persons (e.g. child sexual offender in the family) are tolerated, protected, or defended in the name of such common interest (or shared obligation), that this concept becomes problematic" (p. 961-962).

Finally, Elbedour et al. (2006) say, "family honour can be conceived as a double-edged sword. On the one hand, family honour can protect women because of the dire cost the male perpetrator may pay as a result of violating 
the 'family honour' code. On the other hand, ideology and practices of 'family honour' can place female victims at higher risk of further victimisation as she is afraid of being blamed in the eyes of community and collectivity at large and subsequently for bringing dishonour to her family's name, resulting in repressing her loss and concealing the crime. The mediating/moderating role of family honour in victimisation and exploitation of females has not been fully investigated" (p. 225).

\section{Community resilience as a result of racism and discrimination}

There are other protective factors relevant to ethnic minority communities. For example, Gilligan and Akhtar (2006) note that "(South) Asian families have nurtured their own solidarity and community autonomy in direct response to the hostility of a racist British society" (p. 1370-1371). Thus, experiences of racism as a minority build personal and community resilience. Based on culturally appropriate trauma work in a clinical setting with transgender persons of colour in the US, Singh and McKleroy (2011) similarly say, "mental health providers (should) explore how individuals from historically marginalised communities demonstrate resilience in response to oppression - not to endorse this oppression, but to rather understand how to work with these strengths in healing from these oppressive experiences" (p. 35). More broadly, they call for trauma practitioners to integrate "feminist, multicultural, and social justice principles to radically challenge the traumatology field to focus on the voices of survivors and their strengths for healing, because a deficiency model sustains the oppressive social systems and institutions that allow trauma to occur remain unnamed" (Singh \& McKleroy, 2011, p. 36).

Morris and Balsam (2003) also say, "while lesbian and bisexual women may experience specific risks for victimisation and poor mental health outcomes, it is also likely that they experience strengths or resilience factors due to their sexual orientation that may protect against or moderate the negative mental health consequences of victimisation ... These resiliency factors include: (1) being a part of a community that is more open about discussing personal problems, (2) sharing feminist beliefs about violence and abuse, (3) increased self-esteem and self-confidence associated with being open about one's sexual orientation, and (4) feeling a greater sense of support from friends and seeking out mental health service. Hughes et al. (2001) and Baker (2003) have suggested that strengths may be acquired through the coming out process, such as the ability to cope with distressing emotions, a hostile environment, painful realities, discrimination and prejudice, and being able to better cope with a lack of support from family members and their communities. These resiliency factors or strengths may also be acquired through the development of a secure minority racial, ethnic, or cultural 
identity, as many of these same issues can occur and must be dealt with in the broader society" (cited in Walker, Hernandez, \& Davey, 2012, p. 391-392).

\section{$\underline{\text { Religion }}$}

Religion may also be a culturally specific protective factor. For example, Phasha (2010) says, "an African child is socialised and brought up with exposure to religion, spiritual convictions, and a belief in God or higher power. (Thus), it becomes common to turn to religion when searching for answers to life events as they are understood as God's doings. For this reason, this cultural heritage becomes a major source of strength and a survival strategy when facing a stressful event" (p. 1248).

Haboush and Alyan (2013) say, "devout Muslims frequently cite a reliance on prayer, reading the Qur'an, and consultation with imams as effective coping strategies. Thus, consistent with a collectivist orientation, religious leaders may assist Arab-American families to better support child victims of sexual abuse as well as play an influential role in affirming that Islam does not condone sexual abuse. Related to the worldview that 'life is a test', an external locus of control and belief in divine will, devout Arab-American Muslims (may) also extol patience as a coping strategy, (however), empirical data as to whether children may rely on patience and faith in divine will in coping with child sexual abuse are lacking” (p. 514).

"Hill et al. (1995) studied how African American women react to community violence and concluded that culturally sanctioned mechanisms such as the use of religion and prayer as well as political activism were frequently adopted by African American mothers to cope with the traumatic event of community violence. This study illustrates how female victims of violence appropriate cultural resources to cope with traumatic life events" (cited in Luo, 2000, p. 583).

Finally, Yehuda, Friedman, Rosenbaurn, Labinsky, and Schmeidler (2007) say that "the higher proportion of reported abuse among women who became observant Jews versus those raised observant (suggests that) women who were sexually abused or threatened may be more likely to seek out a more structured and sexually restricted lifestyle" (p. 1704). That is, becoming religious, and seeking greater structure, can provide greater internal locus of control.

Other community-level protective factors 
Finally, there are community-level protective factors. For example, Phasha (2010) notes that in South Africa, school education has been shown to build resilience in survivors of CSA. "Maxwell et al. (2003) found that (rural and suburban high school) students (in the US) with no plans to attend college were over six times more likely to report a history of being sexually assaulted than students who planned to attend college" (cited in Freeman \& Temple, 2010, p. 350). Vermeulen and Greeff (2015) also say, “community-level protective factors that promote family resilience include family involvement in the community, peer relationships, supportive mentors, safe neighbourhoods, access to quality schooling, and health care" (p. 565). Coulton, Crampton, Irwin, Spilsbury, and Korbin (2007) also talk about the importance of neighbourhood when examining the likelihood of child maltreatment. They identified a number of community factors related to the likelihood of reporting child maltreatment in disadvantaged communities. In particular, communities that were more cohesive and with higher social capital tended to have lower reporting rates than similar communities with low cohesion and social capital.

Using empowerment-based approaches

The results additionally highlight the importance of empowerment-based approaches. Compared to the research on strengths-based approaches, Reavey et al. (2006) encouragingly note "the use of more empowerment-based and culturally sensitised models to achieve wellbeing for (South Asian) women who have experienced domestic and sexual abuse (in the UK)" (p. 174). Similarly, Mathews, Abrahams, and Jewkes (2013) say, "counsellors (in South Africa) utilised a feminist approach with a focus on empowering children to be an active participant in their own healing" (p. 650). Finally, Haboush and Alyan (2013) say, "empowerment may also take the form of building on pre-existing cultural concepts, thereby enhancing the likelihood of reporting child sexual abuse; for instance, the Arabic vernacular contains the term awia to indicate a girl or woman who is strong, clever, and capable. Likewise, the task of empowerment is not limited to children but to Arab-American families as well by mobilising the care and protection that is inherent in collectivist systems. Doing so may not only increase the likelihood that children will tell someone about the abuse, it may also discourage other family members from bearing witness to such acts in silence" (p. 514).

\section{A proposed model for Australia}

The literature suggests that there are at least two key approaches to effective service delivery for victims/survivors of CSA from ethnic minority backgrounds. The first is to use an ecological model as a guiding 
framework, and the second is to fundamentally value the victim/survivor as an individual. Together, these help standardise treatment but also offer flexibility to tailor services to unique needs, necessary for effective intervention.

Alaggia (2001) says, "advancing cultural competence and taking culture into consideration are proposed in the assessment and treatment of child sexual abuse, but it is more accurate to say that these (be treated) as cautionary guidelines rather than models of intervention" (p. 43). In this vein, this article proposes an ecological 'model' for service delivery for victims/survivors of CSA from ethnic minority communities in Australia (see Figure 2).

The model places the individual at the centre of intervention, and alerts practitioners to key contextual and interrelated variables that might need to be considered. These have been grouped into one of four tiers: (i) 'traumarelated' factors, (ii) 'service-related' factors, (iii) factors related to belonging to the ethnic minority community in Australia, and (iv) other factors. They draw on all variables identified in the larger study that explored the six themes (but which have not been reported on here for brevity).

This model builds on variables known to affect 'the migrant story', and adapts the principles of cultural competency proposed by Sawrikar $(2017,2014)$ on how to work effectively with ethnic minorities in Western child protection systems more generally. Overall, the practitioner is seen to be as equally responsible for cultural competency as the organisation in which they work (Sawrikar \& Katz, 2014), so that responsibility does not all falsely fall on the shoulders of frontline workers, and their overarching management systems support their work towards cultural competency. This model, however, also emphasises the importance of 'intersectionality' (Crenshaw, 1991) - a movement that emerged during the 1980s and 1990s from Black and Third World feminists to highlight the intersection of several systems of oppression such as racism, sexism, classism, ableism, etc. (Bartolomei, Eckert, \& Pittaway, 2014). This is consistent with several other authors in the field (e.g. Bartolomei et al., 2014; Sokoloff \& Dupont, 2005; Stewart, 2005), and ensures that the victim/survivor from an ethnic minority community is not reduced to their ethnicity, and can decide for themselves what variables they see to be as sources of resilience. For example, 'family cohesion' could be risk factor for one individual and a protective factor for another, making self-determination critical for identifying and implementing factors that aid resilience. 


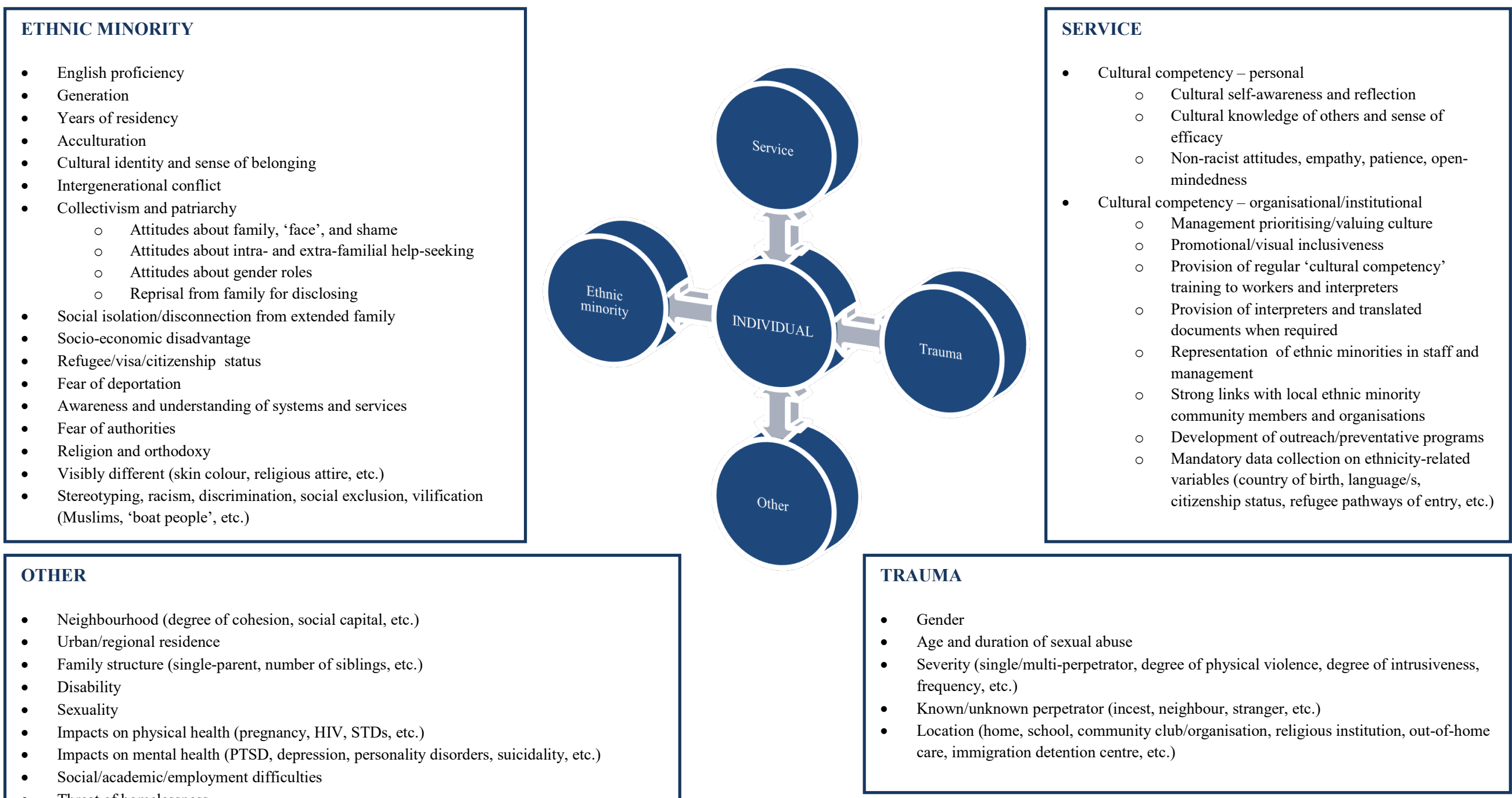


More broadly, "Western methods of dealing with the abuse of women (from) collective societies has prompted researchers and helpers to search for more effective methods of ending female abuse. (That is), practitioners in the field of female victimology (in the context of CSA) have been forced to explore and develop alternative models" (Shalhoub-Kevorkian, 2000, p. 622). The proposed model is one such example, but while it calls for consideration of a feminist framework given that most victims of CSA worldwide are female (Chen, Dunne, \& Han, 2004; Krug, Dahlberg, Mercy, Zwi, \& Lozano, 2002; Thornton \& Veenema, 2015; Wang \& Heppner, 2011), it aims to be relevant for male victims also.

Overall, the model proposed here is really just a comprehensive list of factors that a practitioner would need to be aware of and consider when working with a victim/survivor of CSA from an ethnic minority background in a Western multicultural country like Australia, while conceptually centring the victim/survivor as an individual first and foremost and grouping the many factors under four more simple tiers. It is envisioned that by clearly documenting the full range of variables that would need consideration by a practitioner, standardisation of service delivery is enhanced. The way each variable is addressed or acknowledged in the clinical setting, however, would need to be flexibly responsive to that victim/survivor. Its effectiveness in assisting practitioners' confidence and competence, and in turn enhancing client outcomes, would need to be rigorously evaluated in future research (such as with the use of a longitudinal mixed-method randomised control trial [RCT] study). This would allow for a mapping between specific and effective practice components and approaches, and specific intended client outcomes.

\section{Discussion}

\section{Summary of key findings}

There are currently six theoretical models that may inform service delivery, identified in the literature on CSA and ethnic minority communities. Across them, two overarching principles emerged: value for an ecological approach and value for individuals. These principles have been used to propose a model that could be used in Australia. Such a model can help guide practice and standardise treatment and support in the field, but it also allows for flexibility so that each variable for consideration is identified and self-determined as a risk or protective factor (or even both) by the victim/survivor. The model also values cultural competency, a strengthsbased approach, an empowerment-based approach, intersectionality, and a feminist framework.

\section{Conclusion}


Effective models of service delivery for victims/survivors of CSA in ethnic minority communities in Australia (and elsewhere) are lacking. This reflects a larger gap in research and knowledge on their needs and experiences. The systematic literature review conducted here goes some way in being able to help address this gap. Using the findings, a proposed model has been offered for the national context. Rigorous future evaluative research is required to ultimately examine whether it enhances client outcomes. If such evidence is found, it would have important implications for child protection policy and practice, in terms of how the nexus of cultural diversity and child safety is addressed by statutory authorities and clinicians to whom they make referrals. 


\section{References}

Al-Fayez, G. A., Ohaeri, J. U., \& Gado, O. M. (2012). Prevalence of physical, psychological, and sexual abuse among a nationwide sample of Arab high school students: Association with family characteristics, anxiety, depression, self-esteem, and quality of life. Social Psychiatry and Psychiatric Epidemiology, 47(1), 53-66.

Alaggia, R. (2001). Cultural and religious influences in maternal response to intrafamilial child sexual abuse: Charting new territory for research and treatment. Journal of Child Sexual Abuse, 10(2), 41-60.

Allimant, A., \& Ostapiej-Piatkowski, B., (2011). Studies, (2011). Supporting women from CALD backgrounds who are victims/survivors of sexual violence: Challenges and opportunities for practitioners. Australian Centre for the Study of Sexual Assault (ACSSA) Wrap, No. 9, 1-16, Melbourne: Australian Institute of Family Studies. Australian Institute of Health and Welfare (2017). Child protection Australia 2015-16. Child Welfare series no. 66. Cat. no. CWS 60. Canberra: AIHW.

Baker, C. K., Gleason, K., Naai, R., Mitchell, J., \& Trecker, C. (2013). Increasing knowledge of sexual abuse: A study with elementary school children in Hawai'i. Research on Social Work Practice, 23(2), 167-178.

Baker, S. (2003). Lesbian survivors of childhood sexual abuse: Community, identity, and resilience. Canadian Journal of Community Mental Health, 22(2), 31-45.

Bartolomei, L., Eckert, R., \& Pittaway, E. (2014). “What happens there ... follows us here”: Resettled but still at risk: Refugee women and girls in Australia. Refuge, 30(2), 45-56.

Berry, J. W. (1980). Acculturation as varieties of adaptation. In A. M. Padilla (Ed.), Acculturation: Theory, models, and some new findings. Boulder, $\mathrm{CO}$ : Westview.

Boakye, K. E. (2009). Culture and nondisclosure of child sexual abuse in Ghana: A theoretical and empirical exploration. Law and Social Inquiry, 34(4), 951-979.

Bond, M. H. (2002). Reclaiming the individual from Hofstede's ecological analysis-A 20-year odyssey: Comment on Oyserman et al. (2002). Psychological Bulletin, 128, 73-77.

Bromfield, L. \& Arney, F. (2008). Developing a road map for research: Identifying priorities for a national child protection research agenda: Issues paper. Australian Institute of Family Studies, No 28. 
Bronfenbrenner, U. (1977). Toward an experimental ecology of human development. American Psychologist, $32,513-531$.

Burke, S. \& Paxman, M. (2008). Children and Young people from Non-English speaking backgrounds in Out of Home Care in NSW. Sydney: NSW Department of Community Services.

Cashmore, J., Higgins, D. J., Bromfield, L., \& Scott, D. A. (2006). Recent Australian child protection and out of home care research: What's been done and what needs to be done? Children Australia, 31(2), 4-11.

Chen, J. Q., \& Chen, D. G. (2005). Awareness of child sexual abuse prevention education among parents of Grade 3 elementary school pupils in Fuxin City, China. Health Education Research, 20(5), 540-547.

Chen, J., Dunne, M. P., \& Han, P. (2004). Child sexual abuse in China: A study of adolescents in four provinces. Child Abuse and Neglect, 28(11), 1171-1186.

Chien, M-h. (2013). Identifying key components of an internet information system for Chinese survivors of childhood sexual abuse. Journal of Ethnic and Cultural Diversity in Social Work, 22(3-4), 256-270.

Coohey, C. (2001). The relationship between familism and child maltreatment in Latino and Anglo families. Child Maltreatment, 6, 130-142.

Coulton, C. J., Crampton, D. S., Irwin, M., Spilsbury, J. C., \& Korbin, J. E. (2007). How neighborhoods influence child maltreatment: A review of the literature and alternative pathways. Child Abuse and Neglect, $31(11), 1117-1142$.

Crenshaw, K. (1991). Mapping the margins: Intersectionality, identity politics, and violence against women of color. Stanford Law Review 43(6), 1241-1299.

Elbedour, S., Abu-Bader, S., Onwuegbuzie, A. J., Abu-Rabia, A., \& El-Aassam, S. (2006). The scope of sexual, physical, and psychological abuse in a Bedouin-Arab community of female adolescents: The interplay of racism, urbanization, polygamy, family honor, and the social marginalization of women. Child Abuse and Neglect, 30(3), 215-229.

Fontes, L. (Ed.) (1995). Sexual abuse in nine North American cultures: Treatment and prevention. Newbury Park, CA: Sage. 
Fontes, L. (1993). Considering culture and oppression. Journal of Feminist Family Therapy, 5(1), 25-54.

Freeman, D. H., Jr., \& Temple, J. R. (2010). Social factors associated with history of sexual assault among ethnically diverse adolescents. Journal of Family Violence, 25(3), 349-356.

Fry, A. (2000). Suicidal behaviour in young migrant women. In M. Bashir \& D. Bennett (Eds.), Deeper Dimensions: Culture, Youth and Mental Health, 146-157, Sydney: NSW Transcultural Mental Health Centre.

Futa, K. T., Hsu, E., \& Hansen, D. J. (2001). Child sexual abuse in Asian American families: An examination of cultural factors that influence prevalence, identification, and treatment. Clinical Psychology: Science and Practice, 8(2), 189-209.

Gilligan, P., \& Akhtar, S. (2006). Cultural barriers to the disclosure of child sexual abuse in Asian communities: Listening to what women say. British Journal of Social Work, 36(8), 1361-1377.

Gilligan, P., \& Akhtar, S. (2005). Child sexual abuse among Asian communities: Developing materials to raise awareness in Bradford. Practice (UK), 17(4), 267-284.

Haboush, K. L., \& Alyan, H. (2013). “Who can you tell?” Features of Arab culture that influence conceptualization and treatment of childhood sexual abuse. Journal of Child Sexual Abuse, 22(5), 499-518.

Hill, H. M., Hawkins, S. R., Raposo, M., \& Carr, P. (1995). Relationship between multiple exposures to violence and coping strategies among African-American mothers. Violence and Victims, 10(1), 55-71.

Hofstede (2001): http://www.clearlycultural.com/geert-hofstede-cultural-dimensions/individualism/

Hofstede, G. H. (1980). Culture's consequences: international differences in work-related values. Beverly Hills, Calif: Sage Publications.

Hughes, T. L., Johnson, T. P, \& Wilsnack, S. C. (2001). Sexual assault and alcohol abuse: A comparison of lesbians and heterosexual women. Journal of Substance Abuse, 13, 515-532.

Kanukollu, S. N., \& Mahalingam, R. (2011). The idealized cultural identities model on help-seeking and child sexual abuse: A conceptual model for contextualizing perceptions and experiences of South Asian Americans. Journal of Child Sexual Abuse, 20(2), 218-243. 
Katz, I., \& Connolly, M. (2017). Disproportionality and risk decision-making in child protection. In M.

Connolly (Ed.), Beyond the Risk Paradigm in Child Protection: Current Debates and New Directions. London: Palgrave.

Kenny, M. C., \& Wurtele, S. K. (2012). Preventing childhood sexual abuse: An ecological approach. Journal of Child Sexual Abuse, 21(4), 361-367.

Kenny, M. C., \& McEachern, A. G. (2000). Racial, ethnic, and cultural factors of childhood sexual abuse: A selected review of the literature. Clinical Psychology Review, 20(7), 905-922.

Korbin, J. (2008). Child neglect and abuse across cultures. In E. U. Robinson G, Goodnow J, \& Katz I.

Contexts of Child Development Culture, Policy and Intervention ed. Darwin: Charles Darwin University Press.

Krug, E. G., Dahlberg, L. L., Mercy, J. A., Zwi, A. B., Lozano, R. (2002). World Report on Violence and Health. Geneva: World Health Organisation.

Lesmana, C. B. J., Suryani, L. K., \& Tiliopoulos, N. (2015). Cultural considerations in the treatment of mental illness among sexually abused children and adolescents: The case of Bali, Indonesia. New Directions for Child and Adolescent Development (pp. 109-116).

Li, N., Ahmed, S., \& Zabin, L. S. (2012). Association between childhood sexual abuse and adverse psychological outcomes among youth in Taipei. Journal of Adolescent Health, 50(3, Suppl), S45-S51.

Luo, T-Y. (2000). "Marrying my rapist?!": The cultural trauma among Chinese rape survivors. Gender and Society, 14(4), 581-597.

Mathews, B., Walsh, K., Dunne, M., Katz, I., Arney, F., Higgins, D.,... \& Bates, S. (2016). Scoping study for research into the prevalence of child abuse in Australia: report to the Royal Commission into Institutional Responses to Child Sexual Abuse. Sydney: Social Policy Research Centre, UNSW Australia in partnership with Australian Institute of Family Studies, Queensland University of Technology, and the Australian Centre for Child Protection (University of South Australia).

Mathews, S., Abrahams, N., \& Jewkes, R. (2013). Exploring mental health adjustment of children post sexual assault in South Africa. Journal of Child Sexual Abuse, 22(6), 639-657. 
Maxwell, C. D., Robinson, A. L., \& Post, L. A. (2003). The nature and predictors of sexual victimization and offending among adolescents. Journal of Youth and Adolescence, 32, 465-477.

Meinck, F., Cluver, L. D., Boyes, M. E., \& Mhlongo, E. L. (2015). Risk and protective factors for physical and sexual abuse of children and adolescents in Africa: A review and implications for practice. Trauma, Violence, and Abuse: A Review Journal, 16(1), 81-107.

Mildred, J., \& Plummer, C. A. (2009). Responding to child sexual abuse in the United States and Kenya: Child protection and children's rights. Children and Youth Services Review, 31(6), 601-608.

Moher, D., Liberati, A., Tetzlaff, J., \& Altman, D. G., \& The PRISMA Group. (2009). Preferred Reporting Items for Systematic Reviews and Meta-Analyses: The PRISMA Statement. PLoS Med, 6(7), e1000097.

Morris, J. F., \& Balsam, K. F. (2003). Lesbian and bisexual women’s experiences of victimization: Mental health, revictimization, and sexual identity development. Journal of Lesbian Studies, 7, 67-85.

Nelson, J. K., Dunn, K. M., \& Paradies, Y. (2011). Australian racism and anti-racism: Links to morbidity and belonging. In F. Mansouri \& M. Lobo (Eds.), Migration, Citizenship and Intercultural Relations: Looking Through the Lens of Social Inclusion. Surrey, UK: Ashgate Publishing Ltd.

Nesci, A. (2006). Overcoming barriers to providing domestic violence services for women from culturally and linguistically diverse backgrounds. Parity, 19(2), 44.

O’Hagan. (1999). Culture, cultural identity, and cultural sensitivity in child and family social work. Child and Family Social Work, 4(4), 269-281.

Okur, P., van der Knaap, L. M., \& Bogaerts, S. (2016). Ethnic differences in help-seeking behaviour following child sexual abuse: a multi-method study. Culture, Health and Sexuality, 18(1), 99-111.

Owusu-Bempah, K., \& Howitt, D. (2000). Psychology beyond Western perspectives: London: Wiley-Blackwell. Phasha, T. N. (2010). Educational resilience among African survivors of child sexual abuse in South Africa. Journal of Black Studies, 40(6), 1234-1253. 
Pedraza, S. (2006). Assimilation or transnationalism? Conceptual models of the immigrant experience in America. In R. Mahalingam (Ed.), Cultural Psychology of Immigrants (pp. 33-54). Mahwah, NJ: Lawrence Erlbaum.

Petersen, I., Bhana, A., \& McKay, M. (2005). Sexual violence and youth in South Africa: The need for community-based prevention interventions. Child Abuse and Neglect, 29(11), 1233-1248.

Plummer, C. A., \& Njuguna, W. (2009). Cultural protective and risk factors: Professional perspectives about child sexual abuse in Kenya. Child Abuse and Neglect, 33(8), 524-532.

Poore, G. (2000). The children we sacrifice: A resource book. Silver Spring, MD: Shakti Productions.

Reavey, P., Ahmed, B., \& Majumdar, A. (2006). 'How can we help when she won’t tell us what's wrong?' Professionals working with South Asian women who have experienced sexual abuse. Journal of Community and Applied Social Psychology, 16(3), 171-188.

Sawrikar, P., (2017). Working effectively with ethnic minorities and across cultures in Western child protection systems. UK: Routledge.

Sawrikar, P., \& Hunt, C. J. (2005). The relationship between mental health, cultural identity and cultural values in non-English speaking background (NESB) Australian adolescents. Behaviour Change, 22(2), 97-113.

Sawrikar, P., \& Katz, I. (2008). Enhancing family and relationship service accessibility and delivery to culturally and linguistically diverse (CALD) families in Australia. Issues paper prepared for the Australian Family Relationships Clearinghouse (AFRC), No3.

Sawrikar, P., \& Katz, I. (2009). How useful is the term "Culturally And Linguistically Diverse (CALD)" in the Australian social policy discourse? In Refereed Conference Paper, Australian Social Policy Conference (ASPC) 2009 .

Sawrikar, P., \& Katz, I. (2010). “Only White people can be racist”: What does power have to do with prejudice? Cosmopolitan Civil Societies, 2(1), 80-99.

Sawrikar, P. \& Katz, I. (2014). Recommendations for improving cultural competency when working with ethnic minority families in child protection systems in Australia. Child and Adolescent Social Work, 31(5), $393-417$. 
Sawrikar, P., \& Katz, I. (2017a). Barriers to disclosing child sexual abuse (CSA) in ethnic minority

communities: A review of the literature and implications for practice in Australia. Children and Youth Services Review, 83, 302-315.

Sawrikar, P. \& Katz, I. (2017b). The treatment needs of victims/survivors of child sexual abuse (CSA) from ethnic minority communities: A literature review and suggestions for practice. Children and Youth Services Review, 79, 166-179.

Sawrikar, P., \& Katz, I. (2017c). How aware of child sexual abuse (CSA) are ethnic minority communities? A literature review and suggestions for raising awareness in Australia. Children and Youth Services Review, 81, $246-260$.

Sawrikar, P., \& Katz, I. (2018). Preventing child sexual abuse (CSA) in ethnic minority communities: A literature review and suggestions for practice in Australia. Children and Youth Services Review, 85, 174-186.

Shalhoub-Kevorkian, N. (2000). Blocking her exclusion: A contextually sensitive model of intervention for handling female abuse. Social Service Review, 74(4), 620-634.

Sil, K. H., \& Soo, K. H. (2008). Intra-familial sexual abuse (incest) among Korean adolescents. Family Relations: 21st Century Issues and Challenges (pp. 71-86).

Singh, A. A., \& McKleroy, V. S. (2011). "Just getting out of bed is a revolutionary act": The resilience of transgender people of color who have survived traumatic life events. Traumatology, 17(2), 34-44.

Singh, A. A., Hays, D. G., Chung, Y. B., \& Watson, L. (2010). South Asian immigrant women who have survived child sexual abuse: Resilience and healing. Violence Against Women, 16(4), 444-458.

Singh, A. A. (2009). Helping South Asian immigrant women use resilience strategies in healing from sexual abuse: A call for a culturally relevant model. Women and Therapy, 32(4), 361-376.

Sokoloff, N. J., \& Dupont, I. (2005). Domestic violence at the intersections of race, class, and gender: Challenges and contributions to understanding violence against marginalized women in diverse communities. Violence Against Women, 11(1), 38-64.

Stewart, S. (2005). Suicidality, interpersonal trauma and cultural diversity: A review of the literature. Australian e-Journal for the Advancement of Mental Health (AeJAMH), 4(2), 21p. 
Sulimani-Aidan, Y., \& Benbenishty, R. (2013). Child maltreatment reports in Israel: The intersection between community socioeconomic characteristics and ethnicity. American Journal of Orthopsychiatry, 83(1), 29-36.

Thornton, C. P., \& Veenema, T. G. (2015). Children seeking refuge: A review of the escalating humanitarian crisis of child sexual abuse and HIV/AIDS in Latin America. JANAC: Journal of the Association of Nurses in AIDS Care, 26(4), 432-442.

Tishelman, A. C., \& Geffner, R. (2010). Forensic, cultural, and systems issues in child sexual abuse Cases-Part 1: An Introduction. Journal of Child Sexual Abuse, 19(5), 485-490.

Triandis, H. C. (1995). Individualism and collectivism. Boulder, CO: Westview Press.

Triandis, H. C. (1990). Theoretical concepts that are applicable to the analysis of ethnocentricism. In R. W. Brislin (Ed.), Applied Cross-cultural Psychology. New York: Sage.

Tummala-Narra, P. (2007). Conceptualizing trauma and resilience across diverse contexts: A multicultural perspective. Journal of Aggression, Maltreatment and Trauma, 14(1-2), 33-53.

Usta, J., \& Farver, J. (2010). Child sexual abuse in Lebanon during war and peace. Child: Care, Health and Development, 36(3), 361-368.

Vermeulen, T., \& Greeff, A. P. (2015). Family resilience resources in coping with child sexual abuse in South Africa. Journal of Child Sexual Abuse, 24(5), 555-571.

Walker, M. D., Hernandez, A. M., \& Davey, M. (2012). Childhood sexual abuse and adult sexual identity formation: Intersection of gender, race, and sexual orientation. American Journal of Family Therapy, 40(5), $385-398$.

Wang, Y-W., \& Heppner, P. (2011). A qualitative study of childhood sexual abuse survivors in Taiwan: Toward a transactional and ecological model of coping. Journal of Counseling Psychology, 58(3), 393-409.

Yehuda, R., Friedman, M., Rosenbaurn, T. Y., Labinsky, E., \& Schmeidler, J. (2007). History of past sexual abuse in married observant Jewish women. The American Journal of Psychiatry, 164(11), 1700-1706. 